\title{
Thoracic Vein
}

National Cancer Institute

\section{Source}

National Cancer Institute. Thoracic Vein. NCI Thesaurus. Code C53142.

Either of two veins including the internal and lateral thoracic veins that drain the breasts and chest wall. 